\title{
Los medios digitales todavía no pueden con el papel
}

\author{
Dra. Concha Edo \\ Profesora de Periodismo \\ Universidad Complutense de Madrid
}

\section{RESUMEN}

Internet abrió desde sus primeros pasos unas perspectivas periodísticas que parecían ilimitadas y capaces de modificar en poco tiempo los hábitos de los lectores de todos los países. Pero con los datos en la mano vemos que el ritmo de adaptación es mucho más lento de lo que se pensaba y la red, por el momento, no parece que haya hecho ningún daño al papel, aunque sí que se lo ha hecho a la televisión. El escenario que ahora tenemos a la vista era totalmente imprevisible hace bien poco: Internet se ha convertido en un apoyo para la prensa y se está produciendo un crecimiento constante de las visitas a las páginas web de los grandes diarios, sobre todo para buscar las noticias de última hora.

\section{ABSTRACT}

The Internet opened, from the very beginning, journalistic prospects that seemed limitless and capable of modifying in very little time the habits of readers all over the world. But with statistics at hand, we see that the rhythm of adaptability is much slower than expected and the web, for the moment, does not seem to have done any harm to paper but only to TV. What we have now in sight was absolutely unpredictable a short time ago: the Internet has become a support for the press and visits to the web pages of big newspapers are on the increase, specially to find the latest news.

Palabras claves: Periodismo en Internet/Periodismo digital/Periodismo electrónico/Internet/Prensa digital/Prensa.

Key words: Journalism in the Internet/Digital journalism/Electronic journalism/The Internet/ Digital press/The press.

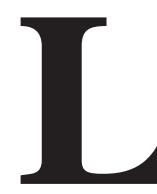

a generalización de Internet y la instalación de las cabeceras con más peso y mayor difusión en el espacio cibernético, hacia la mitad de la década de los noventa, abrieron unas perspectivas periodísticas desconocidas hasta entonces: la utilización del hipertexto, la interactividad con los lectores, el acceso a archivos y bases de datos y, algo más tarde, la instantaneidad en la publicación de las noticias. Todo un conjunto de posibilidades que 
parecían ilimitadas y capaces de modificar en poco tiempo los hábitos de los lectores de todos los países.

Pero después de varios años de adaptación a las nuevas tecnologías, los resultados que está obteniendo la prensa en Internet no responden del todo a las previsiones iniciales, a lo que parecía un cambio inmediato y definitivo en el periodismo: la realidad a día de hoy es que los medios digitales tienen todavía muchas dificultades en el desarrollo de su trabajo y no han resuelto satisfactoriamente sus problemas ${ }^{1}$.

Con los datos en la mano es posible afirmar que, aún cuando no se puede dudar de la importancia del entorno digital, el ritmo de adaptación es mucho más lento de lo que se podía esperar cuando comprobamos que los periódicos de todo el mundo estaban ya en Internet. Parecía que la red iba a ocupar un espacio informativo fijo en la vida de la mayor parte de los ciudadanos en el transcurso de un breve espacio de tiempo, pero han surgido una serie de dificultades cuyas consecuencias vamos a examinar a lo largo de este trabajo.

Para entrar en el análisis de la cuestión vamos a comenzar por estudiar las cifras de difusión de los medios. Después veremos qué ocurre con el aspecto externo y con los contenidos y, finalmente, trataremos de sacar conclusiones y de plantear algunas dudas.

No se va a abordar el desarrollo de la cronología concreta de cada una de las cabeceras y las diferencias cualitativas y cuantitativas de la instalación de sus respectivas páginas web, en nuestro país y en otros, porque ya existen publicaciones que lo muestran, y se parte de la situación actual de cada uno de los medios y de los datos comprobados de las últimas mediciones que se han hecho públicas en 2002 .

\section{Las cifras del papel}

Si comenzamos por fijarnos en los periódicos de nuestro país que superan los 100.000 ejemplares de difusión media y seguimos sus altibajos, podemos establecer en que situación nos encontramos tomando como punto de partida 1995, año en el que los primeros diarios de información general llegaron a la red.

1 Además, los grandes grupos que controlan el sistema informativo internacional siguen siendo los mismos: Time Warner, Disney, Bertelsman, Viacom, News Corporation (Rupert Murdoch), Sony, TCI, Universal, NBC. Y con el agravante de que se ha concentrado más su poder: AOL ha absorbido Time Warner; Vivendi, Universal; Disney, la CBS, y General Electric, la CNBC. En el nivel siguiente -New York Times Co., Hearst, Comcast y Gannet- también se mantienen los nombres. Quizá se han salido de las previsiones los portales de Internet. Pero Yahoo, Terra Lycos, Go.com o Excite no han entrado en directo en la actividad periodística y tienden a ofrecer contenidos de agencias -Reuters, AP, Efe, France Press- o de otros medios, de forma que el monopolio de los grandes, que también tiene portales, sigue intacto. Es verdad que se pueden difundir todo tipo de informaciones e ideas a través de las páginas web personales y foros de discusión, pero ¿quién las lee? Y sobre todo, ¿qué credibilidad tienen?(...) Es evidente que la guerra de Internet no ha terminado, pero también es claro que en los primeros asaltos el monopolio informativo tradicional sólo ha sufrido leves magulladuras (iBrújula, 1 de marzo de 2001). 
La primera observación es de tipo global y evidente: en el año 2002 se venden más papers que cuando no se habían situado en la red que, por el momento, no parece que haya hecho ningún daño al papel.

En estas cifras de difusión de los periódicos vemos que El País, El Mundo, ABC, La Vanguardia, El Periódico de Cataluña, El Correo y La Voz de Galicia sumaban, en los controles de la OJD del año en el que comienzan a contar con ediciones on line, 1.719.465 ejemplares diarios. Y en 2001, el último año controlado, esas mismas cabeceras y La Razón tienen una difusión total de 1.763.086 ejemplares. Los diarios de información general están donde estaban.

Cuadro $n^{\circ} 1$

Diarios de información general que superan los 100.000 ejemplares

\begin{tabular}{|l|l|l|l|l|l|l|}
\hline & $\mathbf{1 9 9 5}$ & 1996 & 1997 & 1998 & 1999 & $\mathbf{2 0 0 1}$ \\
\hline El País & $\mathbf{4 2 0 . 9 3 4}$ & 413.543 & 440.628 & 450.176 & 435.433 & $\mathbf{4 3 3 . 6 1 7}$ \\
\hline El Mundo & $\mathbf{3 0 7 . 6 1 8}$ & 260.616 & 284.519 & 272.299 & 285.303 & $\mathbf{3 1 2 . 3 6 6}$ \\
\hline ABC & $\mathbf{3 2 1 . 5 7 3}$ & 303.010 & 301.054 & 302.013 & 293.053 & $\mathbf{2 7 9 . 0 5 0}$ \\
\hline La Vanguardia & $\mathbf{2 0 3 . 0 2 6}$ & 196.807 & 210.012 & 212.202 & 205.126 & $\mathbf{1 9 1 . 6 7 3}$ \\
\hline El Periódico de C. & $\mathbf{2 1 5 . 5 8 1}$ & 210.793 & 207.772 & 208.070 & 217.607 & $\mathbf{1 8 4 . 2 5 1}$ \\
\hline El Correo & $\mathbf{1 3 7 . 6 4 7}$ & 135.811 & 133.014 & 133.032 & 132.435 & $\mathbf{1 3 1 . 3 8 3}$ \\
\hline La Razón & - & - & - & - & 28.512 & $\mathbf{1 2 2 . 8 9 6}$ \\
\hline La Voz de Galicia & $\mathbf{1 1 3 . 0 8 6}$ & 109.582 & 107.492 & 105.533 & 108.841 & $\mathbf{1 0 7 . 8 5 0}$ \\
\hline TOTAL & $\mathbf{1 . 7 1 9 . 4 6 5}$ & 1.630 .162 & 1.684 .491 & 1.685 .284 & 1.706 .310 & $\mathbf{1 . 7 6 3 . 0 8 6}$ \\
\hline \hline
\end{tabular}

Fuente: OJD/ Elaboración propia

Desglosando los datos:

1. El País y El Mundo ofrecen las mismas cifras que en 1995, aunque parece que el primero se mantiene y el segundo, después de bajar en el 96, sube ligeramente en los últimos controles.

2. ABC cae lentamente, pero sin detenerse por el momento, perdiendo lectores cada año.

3. La Vanguardia y El Periódico de Cataluña, que mantienen en Barcelona una pugna semejante a la de los dos periódicos más importantes de Madrid, tienen altibajos que, en 2002, se saldan a favor de La Vanguardia.

4. El Correo y La Voz de Galicia tienen también altibajos pero más suaves y, tanto uno como otro, han perdido lectores en una proporción pequeña. 
Hay otros estudios internacionales de audiencias que confirman esta situación. El 55 Congreso de la Asociación Mundial de Periódicos (WAN/ AMJ), que reúne a 900 editores de 80 países y se celebró en Brujas (Bélgica) en el mes de mayo de 2002, concluyó sus trabajos con un Informe de Tendencias que reafirma la vigencia del soporte papel y ofrece, entre otros, los siguientes datos ${ }^{2}$ :

- las ventas de diarios en todo el mundo aumentaron un 0,46\% el año pasado, y el $4,8 \%$ si se tienen en cuenta las cifras de los últimos cinco años, aunque los ingresos por publicidad disminuyeron un $7 \%$,

- en la Unión Europea, cinco países vieron aumentar la difusión total de prensa y nueve bajaron sus ventas, con una caída mayor respecto al conjunto europeo en Austria (2,5\%),

- en España las cifras se mantienen estables,

- la bajada de ventas de EEUU ha sido del $0,7 \%$ y la de Japón, del 0,3, mientras que en China han subido un 10,1\%,

- parece que hay una relación directa entre prosperidad y aumento de difusión: con la mejora del nivel de vida baja la difusión de la televisión y sube la de la prensa,

- donde más éxito tienen los periódicos es en Suecia, Finlandia, Noruega, Hong Kong y Japón,

- en los últimos cinco años el dinero de la publicidad aumentó en todos los países de la Unión Europea menos Suecia y Dinamarca.

A todo esto hay que añadir que, en un estudio realizado por esta asociación sobre 429 periódicos ${ }^{3}$, el $50 \%$ aumentó sus beneficios en 2001 y una tercera parte los mantuvieron estables, mientras que sólo el $17 \%$ de los diarios digitales gana dinero.

Otro estudio, realizado por Forrester y denominado The Media Cannibalisation Begins, asegura que el medio que pierde audiencia es la televisión y que el $89 \%$ de los europeos prefiere como fuente de información el periódico impreso en papel ${ }^{4}$.

Y otro dato más: el crecimiento de Internet se frenó en el último ejercicio pero, a la vez, se produjo un aumento considerable del número de visitas a los sites de información de actualidad.

2 El Mundo, 28 de mayo de 2002.

3 Baquía, 30 de mayo de 2002.

4 Baquía, 9 de julio de 2001. 


\section{Un escenario imprevisible}

En este marco, los organizadores del $55^{\circ}$ Congreso llegaron a la conclusión de que Internet no es ni la amenaza que temíamos, ni la oportunidad que habíamos soñado, y aseguran, entre otras cosas, que la red seguirá siendo secundaria con respecto al papel ${ }^{5}$, que es difícil conseguir que se lean las noticias en una pantalla y que la publicidad en Internet sólo representa el $5 \%$ del mercado.

Son muchos los editores que piensan que Internet contribuye a aumentar las ventas en papel, y hay estudios que lo corroboran: en Francia, entre 1999 y 2001 los diarios con páginas web aumentaron su cuota de mercado un $0,6 \%$.

Otro informe hecho público en mayo de 2002, en este caso de Media Planning, asegura que los lectores españoles cuentan con la edición on line, pero prefieren el papel. Así, una media del 22,6 \% de esos lectores de prensa entran en Internet para ver la versión digital, con distintos porcentajes según el medio: el 34,8 \% en Expansión, el 32,7 en El País y el $32 \%$ en El Mundo, entre otroß Y según una encuesta de Newspaper Association of America (NAA), el $75 \%$ de los internautas de Estados Unidos lee periódicos impresos en papel ${ }^{8}$.

Más cercano a nosotros, el Libro blanco de la prensa de la Asociación de Editores de Diarios Españoles (AEDE) ofrece unas propuestas y unos resultados que están en la misma línea: los periódicos, a pesar de las dificultades publicitarias y la leve bajada de lectores prevista para 2002, han salido reforzados de la última década y entre 1991 y 2000 han subido un $11 \%$ la difusión y un $55 \%$ la audiencia ${ }^{9}$. En ese mismo espacio de tiempo la facturación publicitaria subió un $93,3 \%$ y la venta de ejemplares un $72,7 \%$. Con 137 periódicos somos el segundo país de Europa en número de cabeceras, por detrás de Alemania. Pero aun cuando la calidad de la edición es alta, la mayor parte tiene tiradas pequeñas y en número de lectores estamos en una posición de retraso con respecto a otros países europeos de nuestro entorno ${ }^{11}$.

En Estados Unidos, el presidente de The New York Times Company Arthur O. Sulzberger Jr.- afirmaba en el canal público de televisión PBS al finalizar el año 2001 que Internet les proporciona más lectores para el papel ${ }^{12}$. Y

5 Información de Europa Press para Yahoo! Noticias del 29 de mayo de 2002.

6 Baquía, 30 de mayo de 2002.

7 Este trabajo fue recogido por Europa Press.

8 Baquía, 6 de mayo de 2002.

9 Cfr. www.aede.es.

10 Hace unos meses la Fundación de las Cajas de Ahorro Confederadas (FUNCAS) hizo público el informe La lectura en España, en el que se afirma que el $29 \%$ de la población no lee ni libros, ni periódicos, ni revistas. Un dato negativo que, sin embargo, mejora el porcentaje del $42 \%$ que se daba en 1991 (El Mundo, 11 de julio de 2001).

11 El País, 21 de mayo de 2002. Los datos están incluidos en un artículo titulado "Un reto: crear lectores", firmado por José Sanclemente, presidente de la AEDE.

12 IBrújula, 18 de diciembre de 2001. 
posteriormente, en el citado $55^{\circ}$ Congreso Mundial de Periódicos, insistió en la misma idea: el periódico en soporte papel les gusta a los lectores y tiene mucha vida por delante porque se puede doblar, llevar debajo del brazo, meter en la cartera... Y no necesita pilas. La versión digital no es sustituto de nada. Nosotros sabemos que tenemos que estar en todas partes, y estamos.

Se refirió también a que la preocupación inicial de su empresa ante la posibilidad de que la web se llevara lectores era una equivocación porque desde la edición digital el periódico en papel ha conseguido 75.000 nuevos suscriptores $^{13}$. Y recordando el 11 de septiembre de 2001, fecha en la que evitaron las entrevistas tradicionales para dejar que los neoyorquinos expresaran sus sentimientos, dijo que el número de lectores de ese mes fue de 17 millones desde 190 países, que ese día concreto alcanzaron el récord de 21 millones de páginas visitadas en la versión digital, y que a la semana siguiente de los atentados triplicaron la tirada del periódico tradicional en papel.

Personalmente nunca he creído en la muerte súbita del papel, en primer lugar porque la letra impresa no va a desaparecer aunque cambie de soporte y también porque, al menos durante bastantes años, tampoco va a sucumbir el papelprensa que, probablemente, se convertirá en garantía de análisis plural y de credibilidad ante el aluvión informativo que ofrecen tantos portales nacionales e internacionales, cadenas de televisión, emisoras de radio, periódicos, revistas, empresas o páginas individuales presentes en la red. Con lo que sí hay que contar es con que se pueda llegar a utilizar otro tipo de superficie plana semejante al pape $^{14}$-ya existen prototipos, y alguno de ellos se fabrica en serie-, pero con otras características de impresión ${ }^{15}$.

Sin embargo, este escenario que ahora tenemos a la vista era totalmente imprevisible hace bien poco: Internet se ha convertido en un apoyo para la prensa tradicional que, en 2002 y con los controles de difusión sobre la mesa, no tiene en los medios digitales un competidor peligroso aunque no podemos saber durante cuánto tiempo.

13 La Vanguardia, 29 de mayo de 2002.

14 En la NEXPO'99, que se celebró en Las Vegas, se concedió el Premio de Oro 1999 al Diseño Industrial a un invento de IBM para leer la prensa que facilita la conexión a Internet para bajarse textos, gráficos o fotos en un tipo concreto de papel ligero, pero de calidad (El Mundo, 15 de junio de 1999). Es posible, además, que no haya que elegir entre el papel y la pantalla porque desde 1997 el Laboratorio de Medios del Instituto de Tecnología de Massachusetts (MIT) dispone de un prototipo de papel que se podrá rellenar cada día conectándolo a la red y del que habló en Madrid Nicoholas Negroponte (El Mundo, 22 de noviembre de 1997). El principio de la fabricación en serie ha sido hecho realidad en 1999 por las compañías Xerox y E Ink. La primera ha llamado a este nuevo formato Gyricon, y tiene la delgadez del papel y la posibilidad de acceder al mundo digital con una resolución, por ahora, de 400x600 puntos por pulgada. E Ink fabricó el prototipo del MIT con el nombre de Inmedia, con el que se podrá leer el periódico del día apretando un botón (El País, 17 de junio de 1999).

15 C. Edo (2002): Del papel a la pantalla. La prensa en Internet, Comunicación Social, Sevilla, p. 66. 


\section{Los medios no ofrecen grandes novedades}

Aún cuando entre unos medios y otros hay diferencias, no se puede decir que ningún diario haya ofrecido, hasta ahora, grandes novedades: publican lo que ya ofrecían antes -información y servicios- pero con otros sistemas de diseño y de acceso y, en el caso de las noticias de última hora, con más rapidez.

Estamos en una etapa de transición entre lo que han sido los últimos años del siglo XX y lo que es y será el XXI. Y los pasos que están todavía por dar dependen, en buena medida, de los avances más inmediatos de la tecnología y de la generalización de la banda ancha, y pasan por crear un medio totalmente multimedia, con texto, sonido e imágenes fijas y en movimiento, que saque todo el partido a la interactividad y al hipertexto que, hasta ahora, no es lo que más utilizan los lectores de periódicos cuando acceden a las versiones digitales.

Es cierto que se está produciendo un crecimiento constante de las visitas a las páginas web de los grandes diarios, sobre todo para buscar las noticias de última hora y algunos de los diferentes servicios que se ofrecen. Y desde la perspectiva de la empresa sabemos que las dificultades para poner en marcha un medio digital son menores que en el caso de la prensa escrita o audiovisual porque la inversión inicial es menor. Pero ya se ha visto que, si es menos complicado dar el primer paso, conseguir ganar dinero con el periodismo on line es una tarea difícil que todavía no se ha resuelto. Además, existe un problema grave que sale a relucir en cuanto surge la ocasión: el laboral ${ }^{16}$.

$\mathrm{Si}$ pasamos a ocuparnos del aspecto externo de las páginas, la primera observación que se puede hacer es que al informar en Internet el diseño pierde importancia ${ }^{17}$ para dejar paso a la necesidad de enterarse deprisa de lo más importante. Y todo lo que sobra, distrae la atención del lector que va directamente a los titulares. Por otra parte, como destacó en un congreso reciente el profesor Canga ${ }^{18}$, mientras las primeras de los grandes periódicos tienen una fuerte personalidad con la que se identifican los lectores, la home page de esos mismos medios en la red es externamente muy parecida.

Quizá, se podría añadir, para poder alcanzar el máximo de usabilidad. O quizá porque todavía no se sabe exactamente qué es lo que hay que hacer. Como

16 En el II y III Congreso de Periodismo Digital de Huesca, celebrados en febrero de 2001 y enero de 2002, se manifestó una preocupación generalizada por los problemas laborales, tanto en lo que se refiere a las cuestiones económicas y contractuales como en lo que atañe al horario, a la cantidad y al tipo de trabajo y al modo de trabajar.

17 El diseñador Mario García, director de la consultora García Media, se ha referido en distintas ocasiones a esta tendencia.

18 Jesús Canga, catedrático de la Universidad del País Vasco, intervino en el VII Congreso de la Sociedad Española de Periodística, celebrado en Sevilla en marzo de 2002, con la ponencia "Tecnología, diseño periodístico y ediciones digitales. A la búsqueda de nuevas propuestas visuales para medios periodísticos en Internet". 
demostró un estudio del Poynter Institute de Florida ${ }^{19}$, en la lectura de la prensa digital los textos son más importantes que las fotos. Y, sin embargo, ¿no eran multimedia los nuevos medios?, ¿no iban a utilizar un lenguaje nuevo con todos los soportes en acción?

Lo que parecía inminente se ve todavía lejos y ni se ha llegado a crear un medio nuevo, ni existe un lenguaje nuevo que saque todo el partido posible al hipertexto o a la interactividad, ni se cuenta todavía demasiado con las intervenciones de los lectores, ni se ofrece -en este caso por las limitaciones que marca la tecnología- material audiovisual de calidad (...) Todavía no existen los nuevos medios, los medios multimedia, y los que podemos visitar ahora se parecen mucho a los ya conocidos escritos y audiovisuales ${ }^{20}$.

Capítulo aparte merecen los gráficos interactivos, que pueden considerarse como algo característico de la información periodística on line y son un modo de presentar la actualidad que verdaderamente ha sido y es valorado por los lectores. No se puede hablar de esta cuestión sin resaltar su importancia.

En cuanto a las previsiones de futuro para los periódicos, se puede pensar en distintas tendencias: que en cada país se publiquen unos pocos de calidad en papel, con más análisis y opinión, y se ofrezca una información más ligera en Internet, con todo tipo de medios y portales. O bien que se editen cabeceras locales en papel y un pequeño número de diarios digitales mundiales con una oferta informativa universal. Otra posibilidad interesante es hacer periódicos en papel con menos páginas pero con una amplia oferta de conexiones a través de la red: textos más largos, fotos, vídeos, sonido, gráficos, opinión, interpretación e, incluso, las fuentes utilizadas por el periodista que firma la información. El tiempo dirá cual de ellas se va a imponer sobre las demás (...) Quizá esta nueva situación afecte más a las agencias de noticias (...). Pero donde, probablemente, más puede incidir es en el uso de la televisión ${ }^{21}$.

19 Este conocido estudio, hecho público en mayo de 2000, es el resultado de los trabajos llevados a cabo durante cuatro años en Estados Unidos por la Universidad de Stanford y el Poynter Institute. Los investigadores utilizaron microcámaras especiales que se acoplan a la cabeza y un software que registra los movimientos oculares, el tiempo de fijación en los distintos puntos de la pantalla, los clicks del ratón y la navegación de unas páginas a otras. Y confirmaron que lo primero que se lee son los titulares y los sumarios. Otra de las conclusiones es que se leen tres veces más artículos cortos que largos, pero se profundiza en la lectura de los temas que interesan más. Diez años antes el Poynter hizo un estudio semejante con lectores de prensa escrita y la investigación concluyó que los elementos icónicos eran el primer reclamo de lectura.

20 C. Edo, op. cit., p. 110.

21 C. Edo, op. cit., p. 73-74. De acuerdo con diferentes estudios de audiencias, las dos terceras partes de los escolares estadounidenses entre 9 y 17 años prefieren navegar por Internet a ver la televisión (El Mundo, La Vanguardia y La Estrella Digital, 6 de abril de 2000). Y eso tendrá sus consecuencias, más o menos inmediatas: Si en algún lugar ha transcurrido la vida pública del planeta ha sido en la televisión: allí han sido elegidos (y asesinados) presidentes y allí han tenido lugar las guerras, los desórdenes, las grandes competiciones deportivas y los espectáculos musicales. Los mercados de las ciudades han sido ventajosamente reemplazados por la televisión comercial y los propios intelectuales y científicos han tenido que 
Lo cierto es que en 2002, y con los últimos datos de la difusión de los periódicos editados en papel sobre la mesa, no parece existir un peligro inmediato para la prensa diaria de información general que más bien ha tomado Internet como pista de salida para afianzarse. No se han cumplido los pronósticos de Nicholas Negroponte, Bill Gates o Ted Turner, por poner algunos ejemplos, que dieron por sentado que el mundo mediático iba a arrasar el paisaje periodístico escrito cuando, en realidad, no parece haber empezado la ventolera.

El que sí ha acertado por el momento es Jerry Yang, uno de los fundadores de Yahoo -el otro es David Filo- que en 1998 aseguraba tajante: los medios tradicionales no desaparecerán ${ }^{22}$. Y también hizo una previsión bastante atinada el profesor Casasús en su defensa del pape ${ }^{23}$. El profesor Martínez Albertos hizo inicialmente un pronóstico provocador -desaparecerán los periódicos en papel y quizá el periodismo tal como lo entendemos ahora ${ }^{24}$-, pero después lo ha matizado poniendo el énfasis en que, si no se tienen en cuenta unos criterios éticos estrictos, puede desaparecer el periodismo de calidad. Una posibilidad que a todos nos preocupa al comprobar la cantidad de información que ofrece Internet y la necesidad de ofrecer una síntesis periodística adecuada a los lectores.

\section{La información de actualidad en la red}

Y mientras tanto, ¿qué pasa en la red?. Los controles llevados a cabo por las empresas especializadas del sector muestran las preferencias: la participación en chats y en foros, la utilización del correo electrónico, los juegos...¿Es que no interesa la información de actualidad? Pues sí, sí que interesa. Es cierto que el crecimiento de Internet se ha frenado en las últimas mediciones, pero el número de visitas a los sites de los periódicos más conocidos en formato papel sigue creciendo y se alcanzan cantidades verdaderamente millonarias.

salir a esta ágora para exponer sus teorías y sus innovaciones. Frente a esa televisión vertical que reducía a los telespectadores a la pasividad (...), Internet y las redes telemáticas permiten que los espectadores se conviertan en actores, cuando no en agentes sociales. En la medida en que Internet siga siendo una forma de intercomunicación descentralizada, horizontal, multidireccional e interactiva (...) adquirirá una función social cada vez más relevante, convirtiéndose en una alternativa real al imperio que la televisión ha ejercido durante las últimas décadas. Aquí radica una de las cuestiones centrales para el futuro. (J. Echeverría (1996): "Internet y el periodismo electrónico", en Colegio de Periodistas, Barcelona).

22 El Mundo, 11 de noviembre de 1998.

23 Casasús, J. M. y Núñez Ladevèze, L. (1991):Estilo y géneros periodísticos, Ariel, Barcelona. Josep Maria Casasús, catedrático de la Universitat Pompeu Fabra y Defensor del Lector de La Vanguardia, se ha referido en posteriores ocasiones a esta cuestión.

24 José Luis Martínez Albertos, catedrático de la Universidad Complutense, se refiere a esto en M. Albertos, J. L. (1997): El ocaso del periodismo, CIMS, Barcelona. 
Vamos a ver nuestros propios datos y, como en el cuadro anterior, nos fijamos de nuevo en los diarios que tienen mayor difusión en papel para poder establecer comparaciones. Pero con un matiz importante: las cifras más altas las alcanzan los portales, y ningún periódico ha podido superar hasta ahora las visitas que tienen. A pesar de todo, las visitas no dejan de crecer y está claro que hay un público para la prensa en Internet. Lo que no sabemos es en que medida son los mismos que leen el periódico en papel o son lectores nuevos.

\section{Cuadro $\mathrm{n}^{\circ} 2$}

Visitas/ páginas vistas de los diarios en Internet

\begin{tabular}{|c|c|c|c|c|c|c|}
\hline & X-2001 & II-2002 & III-2002 & VII-2002 & VIII-2002 & IX-2002 \\
\hline El País & $\begin{array}{l}10.302 .858 / \\
\mathbf{9 2 . 9 2 6 . 5 7 1}\end{array}$ & $\begin{array}{l}12.340 .167 / \\
\mathbf{1 0 6 . 6 8 0 , 2 7 0}\end{array}$ & - & - & - & - \\
\hline El Mundo & $\begin{array}{l}11.087 .172 / \\
\mathbf{1 0 6 . 1 0 4 . 1 4 7}\end{array}$ & $\begin{array}{l}10.598 .084 / \\
91.176 .976\end{array}$ & $\begin{array}{l}10.971 .289 / \\
\mathbf{9 0 . 1 2 7 . 3 1 0}\end{array}$ & $\begin{array}{l}14.549 .053 \\
\mathbf{1 2 9 . 8 1 0 . 2 8 2}\end{array}$ & & \\
\hline $\mathrm{ABC}$ & $\begin{array}{l}2.958 .086 / \\
\mathbf{3 3 . 9 4 5 . 0 1 3}\end{array}$ & $\begin{array}{l}2.478 .400 / \\
\mathbf{2 4 . 0 7 5 . 1 7 2}\end{array}$ & $\begin{array}{l}2.152 .184 / \\
\mathbf{2 6 . 5 1 9 . 1 0 2}\end{array}$ & $\begin{array}{l}2.267 .519 \\
\mathbf{3 0 . 2 3 8 . 2 9 7}\end{array}$ & $\begin{array}{l}1.844 .688 \\
\mathbf{2 4 . 8 8 1 . 4 6 7}\end{array}$ & \\
\hline La Vanguardia & $\begin{array}{l}1.634 .127 / \\
\mathbf{2 4 . 9 1 3 . 2 5 0}\end{array}$ & $\begin{array}{l}1.786 .479 / \\
17.710 .184\end{array}$ & $\begin{array}{l}1.671 .591 \\
\mathbf{1 3 . 1 4 5 . 2 9 9}\end{array}$ & $\begin{array}{l}2.063 .788 \\
\mathbf{2 0 . 1 3 1 . 2 8 2}\end{array}$ & $\begin{array}{l}1.797 .879 \\
\mathbf{1 9 . 9 6 1 . 7 8 8}\end{array}$ & $\begin{array}{l}2.184 .809 \\
\mathbf{2 1 . 1 0 1 . 5 6 9}\end{array}$ \\
\hline El Periódico de C. & $\begin{array}{l}955.853 / \\
\mathbf{6 . 9 3 5 . 9 4 6}\end{array}$ & $\begin{array}{l}1.026 .968 / \\
7.508 .622\end{array}$ & $\begin{array}{l}1.008 .291 / \\
\mathbf{7 . 8 9 4 . 4 5 9}\end{array}$ & $\begin{array}{l}1.244 .804 \\
\mathbf{9 . 6 0 3 . 1 1 8}\end{array}$ & $\begin{array}{l}1.041 .959 \\
\mathbf{8 . 3 5 8 . 2 2 2}\end{array}$ & $\begin{array}{l}1.399 .083 \\
\mathbf{9 . 5 6 1 . 5 4 5}\end{array}$ \\
\hline El Correo & $\begin{array}{l}729.220 / \\
\mathbf{6 . 3 8 2 . 7 7 3}\end{array}$ & $\begin{array}{l}719.296 / \\
8.663 .301\end{array}$ & $\begin{array}{l}633.832 / \\
\mathbf{7 . 2 4 2 . 4 7 7}\end{array}$ & $\begin{array}{l}707.013 \\
\mathbf{7 . 3 6 5 . 4 5 0}\end{array}$ & $\begin{array}{l}609.684 \\
\mathbf{5 . 6 4 2 . 5 1 9}\end{array}$ & \\
\hline La Voz de Galicia & $\begin{array}{l}317.537 / \\
\mathbf{2 . 3 6 4 . 7 7 4}\end{array}$ & $\begin{array}{l}339.696 / \\
\mathbf{2 . 4 3 9 . 5 8 7}\end{array}$ & $\begin{array}{l}377.232 / \\
\mathbf{2 . 7 5 7 . 7 6 8}\end{array}$ & $\begin{array}{l}440.049 \\
\mathbf{3 . 1 0 1 . 9 2 3}\end{array}$ & $\begin{array}{l}433.653 \\
\mathbf{3 . 1 7 6 . 9 0 5}\end{array}$ & $\begin{array}{l}497.724 \\
\mathbf{3 . 0 9 1 . 2 5 1}\end{array}$ \\
\hline
\end{tabular}

Fuente: OJD/ Elaboración propia 
Dice un estudio reciente de Media Planning que el 22,6\% de los lectores de prensa acceden a las páginas web de los periódicos. Concretando más, el 38,8 $\%$ de los lectores de Expansión, el 32,7 de los de El País, el 32 \% de los de El Mundo y el $11 \%$ de los de El Correo. Y una encuesta de Newspaper Association of America (NAA) asegura que el $75 \%$ de los internautas estadounidenses lee habitualmente.

Y también se puede decir algo sobre los contenidos, sobre lo que diferencia a unos de otros. En su home page de El País, El Mundo, ABC y La Vanguardia, por ejemplo, dicen las mismas cosas pero ofrecen servicios diferentes. Lo peculiar no es completamente periodístico, aunque se informe en cada medio con matices propios. Lo que marca las distancias es lo que se "de más" sobre lo que ofrece en papel, una larga lista de cosas que no son precisamente información periodística.

Las grandes cabeceras en la red, aparte de proporcionar mucha más información y servicios -y, por supuesto la interactividad y el hipertexto que tan poco se utilizan-, tienen su baza principal en las noticias de última hora, en la novedad de dar la información de forma instantánea. Pero en estos momentos, cuando es habitual que los saldos sean negativos y existe la decisión de cobrar por los contenidos, la verdad es que no parece que hayan encontrado un modelo de medio que pueda entusiasmar a las audiencias actuales y futuras.

\section{Algunas conclusiones}

En resumen, los periódicos se siguen vendiendo igual, las visitas que reciben en sus sites buscan sobre todo la actualidad ${ }^{25}$, la red apoya al papel y los medios digitales no son multimedia. Por otra parte, lo más específico de la red, lo que se puede considerar nuevo desde la perspectiva del periodismo, son las weblogs y los boletines por suscripción, y en ambos casos se ofrece puro texto sin imágenes y sin despliegues tecnológicos de ningún tipo.

¿No se iban a crear nuevos medios multimedia con un lenguaje nuevo? La verdad es que, a día de hoy, poco ha cambiado. Faltan algunos años para que los niños que no se han acostumbrado a leer el periódico en papel sean adultos y quizá entonces las pantallas -que serán extraplanas y espectaculares- ganen la batalla. Pero no sabemos hasta que punto ni de que forma. Por otra parte, después de la experiencia de la redacción multimedia y los periodistas "multiuso" del Chicago Tribune y lo que ha costado que haya redacciones separadas, resulta que ahora lo mejor puede ser no separarlas y que la información sea válida para todos los soportes. Es evidente que los grandes problemas son económicos y laborales: no hay negocio y no se trabaja en las condiciones adecuadas.

25 Las noticias de última hora ocupan el primer lugar en las preferencias de los lectores (El Mundo, 18 de diciembre de 2001). 
Quizá resulte -ahora que todos están pensando en cobrar- que el éxito de la información de actualidad en Internet se debe a que es rápida y gratis, a que da lo mismo que los medios convencionales pero mucho antes. Y también son gratis las weblogs y los boletines por suscripción. Para los lectores de prensa Internet es, hoy por hoy, un complemento y los medios no saben cuál es el modelo que necesitan para interesar a las audiencias.

Si se pretende cobrar para subsistir hay que dar algo distinto y mejor. Si no, ya están el papel, la radio y la televisión. El verdadero dilema no es que no se haya encontrado el secreto de un negocio que es difícil, sino que no se sabe todavía cuál es o puede ser la verdadera novedad de la red y no se sabe en que sentido hay que cambiar los esquemas que ya tenemos.

Sólo si la tecnología avanza con rapidez y se ofrece a precios bajos será competitiva la red, con una pantalla plana y ligera, unos ordenadores de bolsillo perfectos o unos teléfonos móviles más completos. De lo que tarde eso depende el futuro periodístico on line. Y de lo que la oferta sepa diferenciarse de lo que ya llega por otras vías: unos medios escritos y audiovisuales que son muy sólidos, ganan mucho dinero y no tienen ninguna intención de dejarse comer el terreno por Internet. 